\title{
Study on Numerical Simulation Method of Gust Response in Time Domain
}

\author{
Jun-Li WANG \\ School of Mechanical Engineering, Shaanxi University of Technology, Hanzhong, P.R. China \\ wjl503@126.com
}

\begin{abstract}
Keywords: Gust Response, Numerical Simulation in Time Domain, Gust to a Step Change in the Angle of Attack Style, One-Minus-Cosine Type Gust, Sin Type Gust.
\end{abstract}

\begin{abstract}
Based on unsteady Navier-Stokes equations, dynamic response in vertical gust flow perturbation is investigated for the aircraft. The grid velocity method was introduced to simulate the gust influence in time domain. First, after applying this method to simulate typical NACA0006 airfoil gust response to a step change in the angle of attack, it is shown that the calculated lift responses of the airfoil agree well with calculated value in reference. This example verified the accuracy of the time domain simulation method. Furthermore, gust responses for the One-Minus-Cosine gust profile and Sin gust profile are analyzed for the flying wing aircraft. It is found that the response of airfoil aerodynamic was obvious during traveling the gust. The research of variation of aerodynamic force under the action of gust response is important to the gust load alleviation and aircraft design.
\end{abstract}

\section{Introduction}

A gust response of wind, also called gust, is a kind of strength determined wind disturbance in atmosphere. The aircraft body will produce additional unsteady aerodynamic force and moment, when the aircraft suffered a gust response. The additional aerodynamic force will resulted in adverse effects on aircraft flight performance. The pilots could control rudder according to the detection data for manned aircraft. This rudder operation can self unload gust load to a certain extent. But for the unmanned aerial vehicle, the procedures set up in advance are required across the gust wind. The set procedures can ensue the gust load reduce system can automatically start and effective operate.

The numerical simulation methods in time domain have been used in aircraft gust load response with the development of computer technology and CFD calculation method. Parameswaran[1] and Singh[2] detailed simulated gust to a step change in the angle of attack on airfoil and wing through applying the perturbation on the flow velocity field. Zaide [3] used unsteady aerodynamic reduced order method for the gust response calculation. Guo-wei YANG [4] and ZHAN Hao [5] from the domestic on the problem of the elastic aircraft gust response are intensive studied.

In the unsteady calculation, the concept of grid speeding is introduced to calculate the effect of gust, and a time-domain gust response computational method are developed. For the two dimensional NACA0006 model, the response curves of aerodynamics are calculated and tested under gust to a step change in the angle of attack. Finally, using the above method, the characteristics of aerodynamics response under the One-minus-cosine gust type and Sin type gust are resolved for a flying wing UAV, and the pressure distribution on flying wing is analyzed under the effect of gusts.

\section{Calculation Model}

\section{Control Equation}

In the inertial Cartesian coordinate system, the three dimension unsteady Navier-Stokes equation as a integral form can be expressed as:

$$
\frac{\partial}{\partial t} \iiint_{\Omega} \bar{Q} d \Omega+\iint_{S}\left(\bar{G}-\bar{Q}_{\vec{q}}\right) \cdot d \vec{S}=\frac{1}{\operatorname{Re}} \iint_{S} \bar{F}^{V} \cdot d \vec{S} .
$$




$$
\begin{gathered}
\bar{Q}=\left[\begin{array}{c}
\rho \\
\rho u \\
\rho v \\
\rho w \\
\rho e_{t}
\end{array}\right], \bar{G}=\left[\begin{array}{ccc}
\rho u & \rho v & \rho w \\
\rho u^{2}+p & \rho v u & \rho w u \\
\rho u v & \rho v^{2}+p & \rho w v \\
\rho u w & \rho v w & \rho w^{2}+p \\
\rho u h_{t} & \rho v h_{t} & \rho w h_{t}
\end{array}\right] \\
\bar{F}^{v}=\left[\begin{array}{ccc}
0 & 0 & 0 \\
\tau_{x x} & \tau_{y x} & \tau_{z x} \\
\tau_{x y} & \tau_{y y} & \tau_{z y} \\
\tau_{x z} & \tau_{y z} & \tau_{z z} \\
\varphi_{x} & \varphi_{y} & \varphi_{z}
\end{array}\right]
\end{gathered}
$$

Eq. 1 is not closed completely, and it needs to introduce the gas thermodynamic relations and Stokes assumption. The fluid control equation of the above integral form is solved numerically, using the finite volume method. The unsteady time derivative term is discretized using the dual time stepping method. The space convection phase is discretized with upwind Roe scheme. The viscous term is discretized using the center discrete format.

\section{Calculation Methods of Gust}

According to thought of the relative movement, gust conditions would be simulated through the introduction of the grid speed. The airfoil was encountered a gust to a step change in the angle of attack in Fig. 1 as an example. The initial state of vehicle is horizontal fly as $V_{\infty}$ speed. The vehicle with a suddenly rising flow effect is equivalent to the airfoil angle of attack increasing $\Delta \alpha\left(\Delta \alpha=\operatorname{arctg}\left(w_{g} / v_{\infty}\right)\right)$. The boundary conditions of angle of attack mutation are directly given to the fluid field calculation of airfoil. Then not only the angle of attack of airfoil is mutated, but also the coupling of angular velocity of pitch will be produced. The calculated response of this state is not the response of the independent of the angle of attack response [6]. And by introducing the concept of grid velocity, the angle of attack mutation of the airfoil equivalent to the entire flow field with a vertical spurt speed. This calculated method will not produce the additional pitching motion. In Fig. 1, the effect of gust effect and the downward movement of whole computational domain grid as $w_{g}$ speed is consistent.

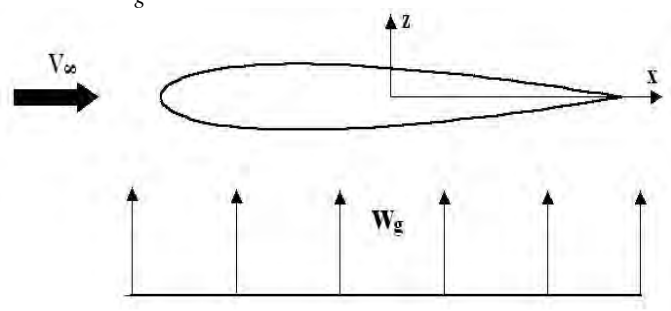

Fig. 1 Sketch of Gust Leading a Step Change in the Angle of Attack

\section{Results and Discussions}

\section{Simulation Method Verification}

For the two dimensional NACA0006 model, the gust response aerodynamics curves under gust to a step change in the angle of attack Style are calculated using time domain mathematical simulation method. The computing grid is $183 \times 43 \mathrm{C}$ type grid with a minimum normal spacing of $1 \times 10^{-5}$. The dual time step method is applied to compute the unsteady flow field. The airfoil flow Maher number of initial state was $M_{\infty}=0.3,0.5,0.65,0.8$. The initial attack angle is $0^{\circ}$. The airfoil angles of attack will produce a 
sudden increase of about $4^{\circ}(0.08$ radian $)$ under the gust to a step change in the angle of attack Style in Fig. $1_{w_{g}} / V_{\infty}=0.08$. The dimensionless time during computation is defined as $s=2 t V_{\infty} / c$.

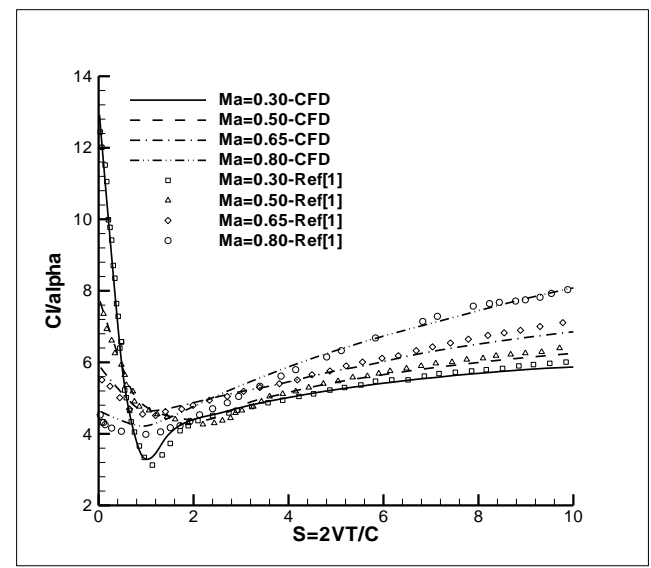

Fig. 2 Comparison of Computing Lift Response for Gust with Ref. [1]

Fig. 2 shows the lift distribution curve of airfoil at different Maher numbers under gust to a step change in the angle of attack Style. The calculation results are compared with the results of Ref. [1].

As can be seen, the two set of results are in good agreement, and the numerical calculation method of gust response in time-domain developed in this paper is reasonable and feasible.

\section{A Flying Wing Aircraft Gust Response Analysis under One-Minus-Cosine Gust Type and Sin Gust Type}

This section takes a flying wing aircraft as the object, and the aerodynamic change characteristics of aircraft is numerical simulated under One-Minus-Cosine gust type and Sin gust type. Two kinds of gust model respectively are as follows.

One minus cosine (1-COS) gust model:

$$
w_{g}=\frac{1}{2} W_{0}[1-\cos (2 \pi x / G G L)]
$$

Sinusoidal (SIN) gust model:

$$
w_{g}=W_{0} \sin (2 \pi x / G G L)
$$

Among them $G G L$ (Gust Gradient Length) is the gust gradient length. The GGL is 25 when simulating the flying wing gust response. The computational grid use multi block structured grid and grid number is 857264. The Maher number is 0.5 and the angle of attack is $0^{\circ}$.The upwind Roe scheme is used for spatial discrimination with S-A turbulence model.

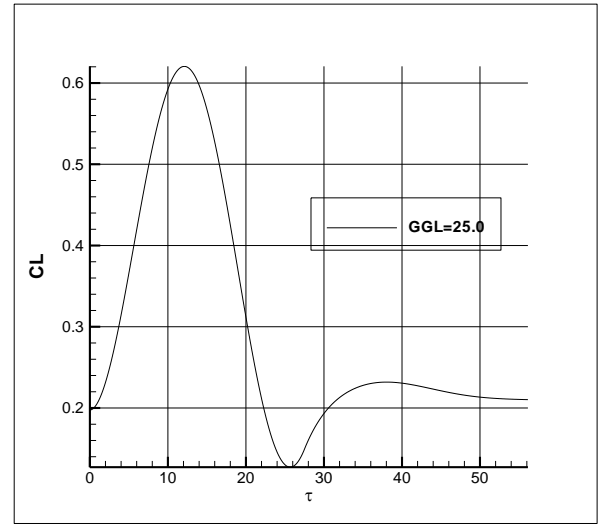

(a)One-minus-cosine gust type

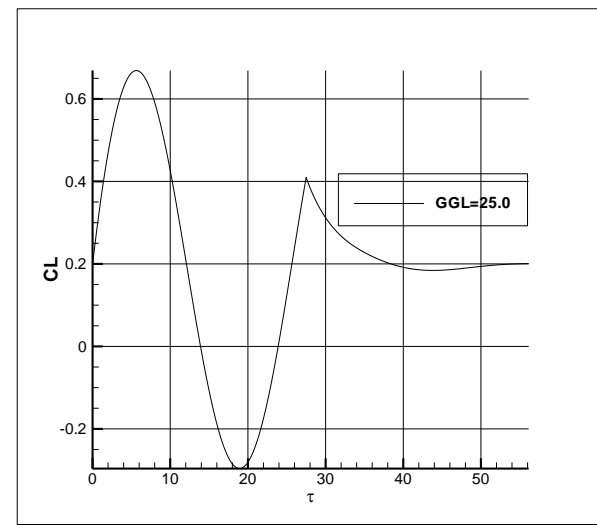

(b)Sin gust type

Fig. 3 Change Curve of Flying Wing Lift Coefficient with Dimensionless Time 
The response curve of flying wing lift coefficient can be seen in Fig. 3 under two kinds of gust model. The lift coefficient produce great changes, and the lift coefficient gradually restored to the initial state with the gust wind disappearing. The response of flying wing lift coefficient is delayed to a certain extent due to the effect of unsteady hysteretic effect. This hysteretic effect makes the lift coefficient response has a certain degree of shake in the gust disappeared.

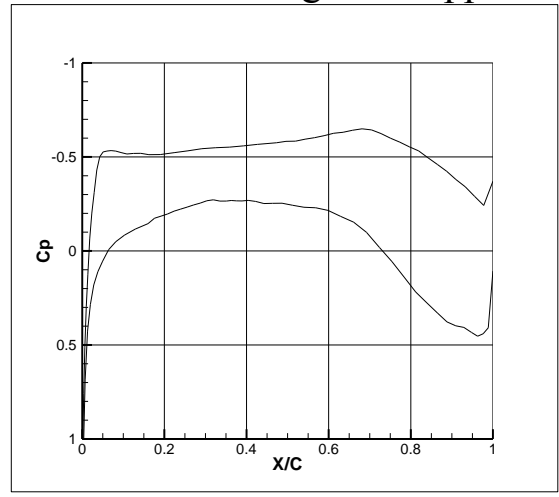

(a) $t=0.0$

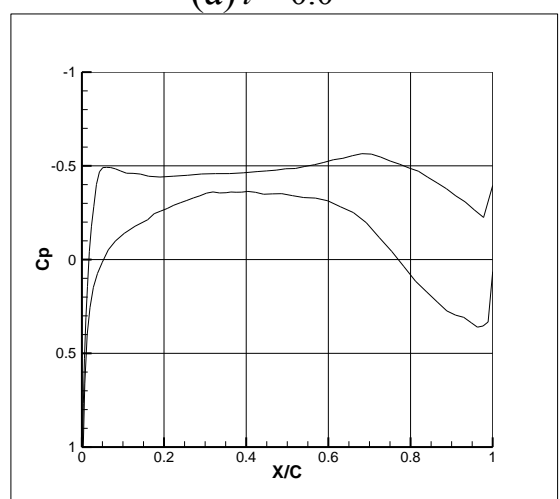

(c) $t=25.5118$

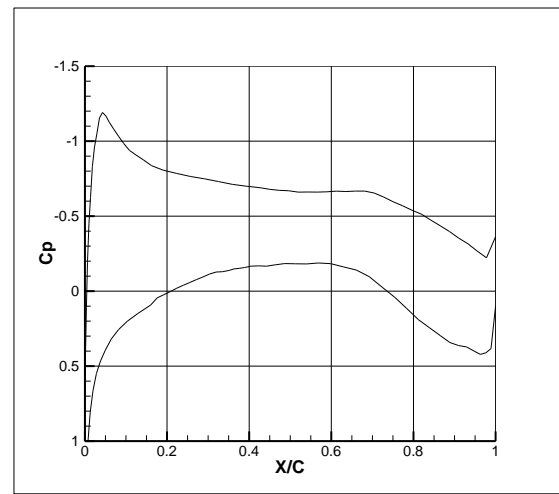

(b) $t=15.3030$

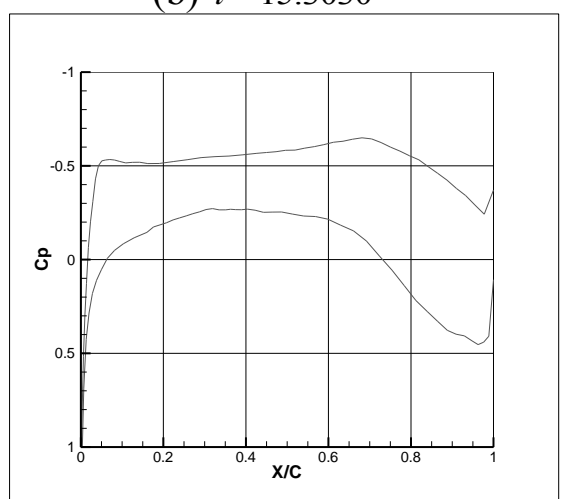

(d) $t=51.0339$

Fig. 4 Pressure Distribution of Flying Wing Spanwise Sections at Various Times

In order to explain the effect reason at the gust to lift coefficient of the flying wing aircraft, the pressure coefficient distribution at various time on the spanwise cross section are analyzed during One-minus-cosine type gust computation. It can be seen in Fig. 4 that the pressure gradient of lower surface enhanced with increasing wind speed. The lift coefficient also increased. With the wind speed continue to increase, the pressure gradient of lower surface no longer enhanced and the suction zone of upper surface increase. At the same time, the lift coefficient continues to increase. The lift coefficient reached the peak until ${ }^{t}$ is 15.3030 . When the gust velocity began to decrease, the pressure gradient of upper surface increased gradually and the suction area decreased. Also the pressure gradient of lower surface gradually decreases; the lift coefficient has a trend to decrease. At time ${ }^{t}$ is 25.5118 , the wind speed reduced to 0 . Due to the unsteady hysteretic effect, the pressure distribution of airfoil is also affected by disturbance. The pressure distribution of airfoil is the same as the distribution of initial state when the dimensionless time ${ }^{t}$ is 51.0339 .

\section{Conclusion}

A time-domain gust response mathematical computation method is developed by introducing the concept of grid speed to calculate the effect of gust. Firstly, the gust response to a step change in the angle of attack style of airfoil was verified by calculation using the developed method. The calculation results are in good agreement with the results in literature. Then the gust response of a flying wing under One-Minus-Cosine type gust and Sin type gust was mathematically simulated. The results showed that the aerodynamic characteristics of aircraft have an obvious change in the gust effect process. The research of the change rule of aerodynamic force under gust effect is significant for gust load alleviation and aircraft design. 


\section{Acknowledgement}

This research was financially supported by Shaanxi University of Technology personnel launch aid project(SLGQD13 (2)-21), the county key technology project in shaanxi province(2012XY-15) and the scientific research project of shananxi province education department in 2014(14JK1160).

\section{References}

[1]Parameswaran, V. and Baeder, J. D., Indicial Aerodynamics in Compressible Flow-Direct Computational Fluid Dynamic Calculations. Journal of Aircraft, Vol. 34, No. 1, 1997, pp. 131-133.

[2]Rajneesh Singh, James D. Baeder. Direct Calculation of Three-Dimensional Indicial Lift Response Using Computational Fluid Dynamics. Journal of Aircraft, 1997, 34 (4):465-471.

[3]Avi Zaide, Daniella Raveh. Numerical Simulation and Reduced-Order Modeling of Airfoil Gust Response. AIAA paper 2005-5128.

[4]Guowei Yang, Shigeru Obayashi. Numerical Analyses of Discrete Gust Response for an Aircraft. Journal of Aircraft. 2004, 41(6):1353-1359.

[5]ZHAN Hao, Wei-qi QIAN. Numerical simulation on gust response of elastic wing. Chinese Journal of Computational Mechanics, 2009, 26(2):270-274.

[6]ZHAN Hao, Wei-qi QIAN. Numerical simulation of gust response for airfoil and wing. ACTA AERODYNAMICA SINICA, 2007, 25(4):531-536. 